\title{
MiR-200-3p Is Potentially Involved in Cell Cycle Arrest by Regulating Cyclin A during Aestivation in Apostichopus japonicus
}

\author{
Shanshan Wang ${ }^{1}$, Muyan Chen ${ }^{1, *}$, Yingchao Yin ${ }^{1}$ and Kenneth B. Storey ${ }^{2}$ \\ 1 Key Laboratory of Mariculture (Ocean University of China), Ministry of Education, Ocean University of \\ China, Qingdao 266003, China \\ 2 Institute of Biochemistry, Carleton University, Ottawa, ON K1S 5B6, Canada \\ * Correspondence: chenmuyan@ouc.edu.cn
}

Received: 9 July 2019; Accepted: 4 August 2019; Published: 6 August 2019

\begin{abstract}
The sea cucumber (Apostichopus japonicus) has become a good model organism for studying environmentally induced aestivation in marine invertebrates. We hypothesized that mechanisms that arrest energy-expensive cell cycle activity would contribute significantly to establishing the hypometabolic state during aestivation. Cyclin A is a core and particularly interesting cell cycle regulator that functions in both the $S$ phase and in mitosis. In the present study, negative relationships between miR-200-3p and $A j C A$ expressions were detected at both the transcriptional and the translational levels during aestivation in A. japonicus. Dual-luciferase reporter assays confirmed the targeted location of the miR-200-3p binding site within the $A j C A$ gene transcript. Furthermore, gain- and loss-of-function experiments were conducted in vivo with sea cucumbers to verify the interaction between miR-200-3p and $A j C A$ in intestine tissue by qRT-PCR and Western blotting. The results show that the overexpression of miR-200-3p mimics suppressed $A j C A$ transcript levels and translated protein production, whereas transfection with a miR-200-3p inhibitor enhanced both $A j C A$ mRNA and AjCA protein in A. japonicus intestine. Our findings suggested a potential mechanism that reversibly arrests cell cycle progression during aestivation, which may center on miR-200-3p inhibitory control over the translation of cyclin A mRNA transcripts.
\end{abstract}

Keywords: cell cycle; miR-200-3p; AjCA; Apostichopus japonicus; aestivation

\section{Introduction}

The sea cucumber, Apostichopus japonicus, is an important food and medicinal species with high commercial value that is widely grown and cultured in Asia [1]. This species is a model marine aestivator that endures a prolonged torpor annually (lasting up to 100 days), brought about by elevated sea water temperatures, and is accompanied by a series of typical characteristics of hypometabolism such as metabolic rate depression, organ atrophy, body mass reduction, energy redistribution and immune system modification [2,3]. The period of aestivation shortens the growing cycle of $A$. japonicus and results in economic losses for the sea cucumber aquaculture industry. Research on the mechanisms involved in sea cucumber aestivation has surged in the past two decades and has shown that sea cucumbers have developed various physiological and biochemical strategies to overcome estivation-associated stresses. These strategies facilitate survival over prolonged periods of dormancy, as seen among other aestivators [2,4,5], and, after arousal from aestivation, promote the recovery of all functions back to normal. With the coming of the omics era, the molecular mechanisms underlying the physiological and biochemical strategies that support aestivation are gradually being unraveled. Over numerous studies, we have confirmed that long-term aestivation in sea cucumbers is supported by molecular 
modifications that include the global suppression of transcription and translation, reversible protein phosphorylation and epigenetic modifications [5-11]. The types of regulatory mechanisms that are best suited for long-term torpor survival must be easily induced, readily reversed, and have a low energy demand [12]. Since their early recognition [13], microRNAs (miRNAs) have become known as one of the most important post-transcriptional regulatory mechanisms, fulfilling the requirements for a mechanism that could be key to aestivation control. MiRNAs suppress gene expression by binding to gene transcripts and targeting them either for degradation or storage. In situations when animals are responding to extreme environmental stress (e.g., estivation, hibernation, anoxia and freezing) [14], select miRNAs are known to be involved in up- or down-regulating various cellular processes such as apoptosis, cell cycle progression, signal transduction, and lipid metabolism [15-21]. However, until now, miRNA-targeted regulatory mechanisms supporting aestivation in marine invertebrates have rarely been documented. A prior limited study by our group suggested the potential contribution of miR-200-3p in the control of fatty acid metabolism by regulating the expression of the AjEHHADH gene (encoding enoyl-CoA hydratase and 3-hydroxyacyl-CoA dehydrogenase) during aestivation in sea cucumbers [11].

The suppression of energy-expensive cellular processes is the common survival strategy during hypometabolism. For example, the cell cycle, one of the most energy-expensive costs for cells, is typically strongly suppressed or arrested in tissues as one component of overall metabolic depression [22]. It has been reported that cell cycle arrest is crucial for ATP (adenosine-triphosphate) homeostasis and the conservation of fuel stores in hibernating thirteen-lined ground squirrels, Ictidomys tridecemlineatus [23], and to achieve long-term anoxia endurance in the turtle Trachemys scripta elegans [22]. However, little is known about the epigenetic regulation mechanisms involved in cell cycle arrest operating during hypometabolism, in particular, for aestivation in sea cucumbers.

Cyclin A was the first cyclin identified and subsequently cloned in organisms [24]. Cyclin A is a pivotal regulator of cell cycle progression at the onset of both DNA replication and mitosis [25]. The mRNA and protein products of Cyclin A begin to accumulate in the late G1 stage and peak in the $S$ phase [26]. Many studies have reported a role for miRNA in regulating the proliferative state of human tumor cells by targeting cyclins [27-30]. However, the potential roles of miRNA in states of hypometabolism where proliferation is suppressed, and the roles of Cyclin A and miRNA-targeted regulatory mechanisms in sea cucumbers remain unknown.

Our present work aimed to study the potential role of miR-200-3p in inhibiting cell proliferation during estivation in the intestine of $A$. japonicus by targeting cyclin A gene expression. We focus on the intestine in the present study because it is the major site responsible for the strong metabolic rate depression seen under deep aestivating conditions, and the global miRNA expression profile of this organ has also been constructed using Solexa deep sequencing technology in our previous study [5]. We initially characterized changes in the transcription and translation of Cyclin A in the intestine of $A$. japonicus over the whole period of aestivation. In vivo and in vitro analyses of the functional relationship between miR-200-3p and Cyclin A were also assessed. The results yielded a picture of the control of Cyclin A in sea cucumber tissues and its adaptive regulation in aestivation-induced post-transcriptional suppression.

\section{Materials and Methods}

\subsection{Animals}

Adult sea cucumbers (males and females) (A. japonicus), $90 \pm 10 \mathrm{~g}$, were collected from the coast of Qingdao (Jiaozhou Bay of the Yellow Sea, China). Three stages of $A$. japonicus were sampled and dissected right after capture. Non-aestivating (NA) animals were sampled on 12 May, when the seawater temperature was about $15^{\circ} \mathrm{C}$ and sea cucumbers had already recovered fully from aestivation. Animals in deep-aestivation (DA) were sampled on 21 July, when seawater temperature was above $28{ }^{\circ} \mathrm{C}$. These animals were sampled after about 15 days of continuous aestivation as indicated by 
the cessation of feeding and locomotion, and the degeneration of the intestine into a very thin and small string (about $2 \mathrm{~mm}$ ). Animals in arousal from aestivation (AA) were sampled on 12 October, when seawater temperature had decreased back to $15^{\circ} \mathrm{C}$. These animals were recently aroused from aestivation, and moving and feeding were observed. For each stage (NA, DA and AA), 10 individuals were sacrificed and intestine tissues were dissected, cleared of contents and flash frozen in liquid nitrogen. All samples were kept at $-80^{\circ} \mathrm{C}$ for later analysis. The study protocol was approved by the Experimental Animal Ethics Committee of the Ocean University of China.

\subsection{Prediction of the miR-200-3p Target}

TargetScan 5.2 and Miranda 3.3a software was used to predict the gene targets of miR-200-3p action, based on our previous transcriptome and proteome databases for A. japonicus [8,9], following the criterion in the seed region-no mismatch between 2-8 nt on the end of miRNA (7mer-m8), and all the parameters were set as default values. Potential targets of miR-200-3p were also predicted using the Miranda toolbox, with parameters set up as follows: single-residue pair score less than a threshold value of 90 and a minimum free energy lower than $-10 \mathrm{kcal} / \mathrm{mol}$. Combining these analyses, cyclin $A$ was selected as a strong candidate target gene of miR-200-3p.

\subsection{RNA Extraction, Cyclin A cDNA Cloning and Sequence Analysis}

Total RNA was isolated from intestinal tissues of A. japonicus using Trizol (TransGen Biotech, Beijing, China, Cat No. J20921) according to the manufacturer's instructions. Intestinal tissues of A. japonicus were ground into powder using liquid nitrogen, and 50 to $100 \mathrm{mg}$ were added to $1.5 \mathrm{~mL}$ centrifuge tubes with $1 \mathrm{~mL}$ Trizol, then centrifuged at $14,000 \mathrm{rpm}$ at $4{ }^{\circ} \mathrm{C}$ for $10 \mathrm{~min}$. The supernatant was moved to a new tube and the Trizol extraction was repeated on the pellet. Then, the second supernatant was mixed with the first and $200 \mu \mathrm{L}$ chloroform was added in a new centrifuge tube (Sinopharm Chemical Reagent Beijing, Beijing, China, Cat No. 10006818). After mixing for $30 \mathrm{~s}$ and resting for $3 \mathrm{~min}$, tubes were then centrifuged at $14,000 \mathrm{rpm}$ at $4{ }^{\circ} \mathrm{C}$ for $15 \mathrm{~min}$, and the supernatant was removed. This step was also repeated twice, and then the final supernatants were combined and mixed with the same volume of isopropanol (Sinopharm Chemical Reagent Beijing, Beijing, China, Cat No. 80109218) in a new centrifuge tube at $-20{ }^{\circ} \mathrm{C}$ for $10 \mathrm{~min}$, then centrifuged at $14,000 \mathrm{rpm}$ at $4{ }^{\circ} \mathrm{C}$ for $8 \mathrm{~min}$. Supernatants were discarded and pellets were dried at room temperature for $10 \mathrm{~min}$, followed by addition of 20-50 $\mu \mathrm{L}$ RNase-free Water (Takara, Japan, Cat No. 9012) to dissolve RNA for $30 \mathrm{~min}$. RNA concentration and quality were determined using a NanoDrop 2000 and 1\% agarose gel electrophoresis, respectively (Thermo, Waltham, MA, USA).

The full-length cDNA sequences were cloned using a SMARTer ${ }^{\circledR}$ RACE (rapid amplification of cDNA ends) 5'/3' Kit (Clontech, Mountain View, CA, USA, Cat No. 634858) following the manufacturer's instructions. The primer sets for cyclin $A 5^{\prime} / 3^{\prime}$ RACE are listed in Table 1 . Polymerase chain reaction (PCR) amplification was carried out using Tks Gflex ${ }^{\mathrm{TM}}$ DNA Polymerase (Takara, Kusatsu, Japan, Cat No. R060A) in a volume of $50 \mu \mathrm{L}$. PCR was performed under the following conditions: $95^{\circ} \mathrm{C}$ for $1 \mathrm{~min} ; 5$ cycles of $94{ }^{\circ} \mathrm{C}$ for $30 \mathrm{~s}, 70{ }^{\circ} \mathrm{C}$ for $30 \mathrm{~s}$, and $72{ }^{\circ} \mathrm{C}$ for $3 \mathrm{~min} ; 5$ cycles of $94{ }^{\circ} \mathrm{C}$ for $30 \mathrm{~s}, 68^{\circ} \mathrm{C}$ for $30 \mathrm{~s}$, and $72{ }^{\circ} \mathrm{C}$ for $3 \mathrm{~min} ; 5$ cycles of $94{ }^{\circ} \mathrm{C}$ for $30 \mathrm{~s}, 66^{\circ} \mathrm{C}$ for $30 \mathrm{~s}$, and $72{ }^{\circ} \mathrm{C}$ for $3 \mathrm{~min} ; 20$ cycles of $94^{\circ} \mathrm{C}$ for $30 \mathrm{~s}, 64^{\circ} \mathrm{C}$ for $30 \mathrm{~s}$, and $72{ }^{\circ} \mathrm{C}$ for $3 \mathrm{~min}$, followed by a final cycle of $72{ }^{\circ} \mathrm{C}$ for $10 \mathrm{~min}$. The PCR products were eluted from a $2 \%$ agarose gel using the NucleoSpin Gel and PCR Clean-Up Kit (Clontech, Mountain View, CA, USA, Cat No. 740609.10) following the manufacturer's instructions, then cloned into the pMD19-T vector (Takara, Japan, Cat No. 6013) and transformed into E.coli DH5 $\alpha$ Competent Cells (Takara, Kusatsu, Japan, Cat No. 9057) following the manufacturer's instructions. Transformed cells were cultured overnight in Luria-Bertani (LB) agar plates containing $100 \mu \mathrm{g} / \mathrm{mL}$ ampicillin. White clones were selected and cultured in SOC medium (Hopebio, China, Cat No. HBDC002) containing $100 \mu \mathrm{g} / \mathrm{mL}$ ampicillin for $12 \mathrm{~h}$ at $37^{\circ} \mathrm{C}$. Positive recombinant clones were sequenced by BGI Genomics (Qingdao, China). The sequences were analyzed and assembled using DNAStar software (DNAStar Inc., USA) to obtain the full-length cDNA and identify the open reading 
frame (ORF). The deduced amino acid sequence of $A j C A$ was analyzed using an on-site program (http://www.bio-soft.net/sms/index.html). The functional sites or domains in the amino acid sequence were predicted using Interpro (http://www.ebi.ac.uk/interpro/) software.

\subsection{Expression Level Analysis of miR-200-3p and AjCA}

Total RNA was isolated as described above from A. japonicus intestine tissues of the NA, DA and AA groups, and the good quality of the RNA was indicted by a 260/280 ratio of 1.8 2.1. For qRT-PCR analysis of miR-200-3p, first-strand cDNA was synthesized using the Mir-X miRNA First-Strand Synthesis Kit (Takara, Kusatsu, Japan, Cat No. 638313), and then qRT-PCR was performed using the SYBR ${ }^{\circledR}$ Premix Ex Taq ${ }^{\mathrm{TM}}$ II Kit (Takara, Kusatsu, Japan, Cat No. RR820) with the mRQ 3' primer supplied with the kit and miR-200-3p specific primer (Table 1). The $5.8 \mathrm{~s}$ rRNA was selected as the internal control (Table 1) [11]. For qRT-PCR analysis of $A j C A$, the first-strand cDNA was synthesized using a PrimeScript ${ }^{\mathrm{TM}}$ RT reagent Kit with gDNA Eraser (Takara, Kusatsu, Japan, Cat No. RR047A), and qRT-PCR was performed using the SYBR ${ }^{\circledR}$ Premix Ex Taq ${ }^{\mathrm{TM}}$ Kit (Takara, Kusatsu, Japan, Cat No. RR420) with the AjCA specific primer pairs (Table 1). $\beta$-tubulin (TBB, PIK103) was selected as an internal control and had been previously validated as being unchanged during $A$. japonicus aestivation in an initial study of reference gene stability [31]. $\beta$-actin (ACTB, PIK61412.1) was also selected as another internal control, which was mainly to ensure the constant expression of an internal control and made the results more stable and accurate. The expression levels of miR-200-3p and AjCA were detected using the StepOnePlus system (ABI Inc., Foster City, CA, USA). Each sample was run in three technical triplicates. Melting-curve analysis of the amplification products was performed at the end of the PCR to confirm specificity.

Table 1. Primer sequences information.

\begin{tabular}{|c|c|c|c|}
\hline & Name & Primer Sequences $\left(5^{\prime}-3^{\prime}\right)$ & Location \\
\hline \multirow[t]{2}{*}{ RACE } & AjCA-F1 & AGCTGGTCGGAACGGCAAGTGCA & $1269-1291$ \\
\hline & AjCA-R1 & GATTGTCGGGACAGCCAGATCAAAGGAC & $1414-1441$ \\
\hline \multirow[t]{10}{*}{ qRT-PCR } & miR-200-3p-F & TAATACTGTCTGGTGATGATG & \\
\hline & miR-200-3p-R & mRQ 3'primer & \\
\hline & $5.8 \mathrm{~s}-\mathrm{F}$ & ATCACTCGGCTCGTGCGTC & \\
\hline & $5.8 \mathrm{~s}-\mathrm{R}$ & GCCATTTGCGTTCGAATAAGT & \\
\hline & AjCA-RT-F & TATCAAGGCCAGCGACGAAGGAG & $1461-1448$ \\
\hline & AjCA-RT-R & GGAGATGCAATGTGTGTCGAGCC & $1594-1616$ \\
\hline & $\beta$-actin-F & AAGGTTATGCTCTTCCTCACGCT & \\
\hline & $\beta$-actin-R & GATGTCACGGACGATTTCACG & \\
\hline & $\beta$-Tubulin-F & GAAAGCCTTACGACGGAACA & \\
\hline & $\beta$-Tubulin-R & CACCACGTGGACTCAAAATG & \\
\hline \multirow[t]{6}{*}{ In vivo } & miR-200-3p mimics-F & UAAUACUGUCUGGUGAUGAUG & \\
\hline & miR-200-3p mimics- $R$ & UCAUCACCAGACAGUAUUAUU & \\
\hline & miR-200-3p mimics-NC-F & GAGAUGUUCAAUCGGGUAUUU & \\
\hline & miR-200-3p mimics-NC-R & AUACCCGAUUGAACAUCUCUU & \\
\hline & miR-200-3p inhibitor & CAUCAUCACCAGACAGUAUUA & \\
\hline & miR-200-3p inhibitor-NC & GAAUUACAUGCACCACUCAAU & \\
\hline \multirow[t]{4}{*}{ Dual-luciferase } & $A j C A-\mathrm{WT}-\mathrm{F}$ & GCGGCTCGAGGACATCTCCAAATTAAAGGA & $1806-1836$ \\
\hline & AjCA-WT-R & AATGCGGCCGCGTAACACTTATGTACAAATG & 3010-3029 \\
\hline & AjCA-MUT-F & TGAATCTTGTCATAAACAAGAACGGGTTTTTAC & $2164-2197$ \\
\hline & AjCA-MUT-R & GTTCTTGTTTATGACAAGATTCATTTTAAAAAC & $2155-2187$ \\
\hline
\end{tabular}

Protein extraction and Western blot analysis were performed as previously described [11]. In brief, total protein was extracted from intestine tissues using Cell lysis buffer (Beyotime, Shanghai, China, Cat No. P0013) following the manufacturer's instructions. Protein samples were boiled for $5 \mathrm{~min}$ in SDS sample buffer and then separated on 10\% SDS-PAGE gels, followed by transfer to polyvinylidene fluoride (PVDF) membranes (Millipore, Bedford, MA, USA, Cat No. IPVH00010). Then, the membranes were incubated with $A j C A$ antibody (1:1000, prepared by GenScript, NanKing, China) or $\beta$-tubulin antibody (1:1000, CST, Cat No. 2146S) overnight at $4{ }^{\circ} \mathrm{C}$, after blocking with $5 \%$ non-fat milk in TBS-Tween 20 buffer (TBST) for $2 \mathrm{~h}$ at $30^{\circ} \mathrm{C}$. After washing with TBST for five times $(5 \mathrm{~min}$ 
each), the membranes were incubated with goat anti-rabbit IgG (Immunoglobulin G) labeled with HRP (1:10,000; Cat No. 7074S; CST, Danvers, MA, USA) for 2 h at room temperature. Five more washes with TBST followed, and then bound antibodies were detected with an ECL substrate kit (Beyotime, Shanghai, China, Cat No. P0018) according to the manufacturer's instructions.

\subsection{Dual-Luciferase Reporter Assays}

The experiment was conducted as described previously [11]. In brief, both wild-type (WT) and mutant (Mut) segments of the AjCA 3'UTR (untranslated region) (about 500 bp before and after the binding sites) were cloned into a pmiR-RB-REPORT ${ }^{\mathrm{TM}}$ luciferase reporter vector (Ribobio, Guangzhou, China). The selected primers are listed in Table 1. The 293T cells were plated onto 96-well white plates $24 \mathrm{~h}$ before transfection. Plasmids constructed with pmiR-RB-REPORT ${ }^{\mathrm{TM}}$ vectors were cotransfected with a control Renilla luciferase plasmid (pRL-CMV) (Promega, Madison, WI, USA). The ratio of experimental plasmid to control plasmid was 5:1. Then vectors with 3'UTR of $A j C A$ (WT and Mut) were cotransfected with miR-200-3p mimics or a negative control. Luciferase assays were performed using the Dual-Luciferase Reporter Assay System (Promega, USA). In brief, $48 \mathrm{~h}$ after transfection, cell lysates were prepared by incubating with $1 \times$ passive lysis buffer for $15 \mathrm{~min}$ at room temperature. Cell lysates were transferred to 96-well plates, analyzed using the luciferase dual reporter assay kit (Promega, USA) and measured with a Veritas microplate luminometer (Turner BioSystems, Sunnyvale, CA, USA). An over $30 \%$ decrease in the fluorescence signal was considered to be confirmation that cyclin A was targeted by miR-200-3p.

\subsection{Loss and Gain-Functional Analysis of miR-200-3p In Vivo}

The miR-200-3p mimics, inhibitor and negative control were synthesized at GenePharma (Shanghai, China) and are shown in Table 1. MiR-200-3p mimics, inhibitor and corresponding negative controls were dissolved into RNase-free water to obtain a working solution of $20 \mathrm{mM}$. Lipo6000 ${ }^{\mathrm{TM}}$ Transfection Reagents (Beyotime, China) were used following the manufacturer's instructions. Aliquots of $300 \mu \mathrm{L}$ mimics or inhibitor as well as a corresponding negative control were mixed with equal volume of Lipo6000 ${ }^{\mathrm{TM}}$ Transfection Reagents, then mixed with $2400 \mu \mathrm{L}$ of PBS (phosphate buffered saline) to serve as the working solution. Twenty-four sea cucumbers at the NA stage (100 $\pm 10 \mathrm{~g})$ were injected with $100 \mu \mathrm{L}$ of the miR-200-3p mimics or inhibitor or negative control mixes, and a booster injection was given after $24 \mathrm{~h}$. The treated and control animals were sacrificed, and intestine tissues were collected after a further $24 \mathrm{~h}$ and stored in liquid nitrogen for further study.

\subsection{Statistics}

The $2^{-\triangle \Delta \mathrm{Ct}}$ method was used to analyze the expression levels of both miR-200-3p and AjCA mRNA, and the data were subjected to one-way analysis of variance (ANOVA) followed by a Tukey's post hoc test using SPSS 17.0 software (Chicago, IL, USA). All results are given as mean \pm S.E. $(n=5)$. Western blotting bands were quantified using Image-Pro Plus 6.0 software (Media Cybernetics Inc., Rockville, MD, USA) and were also subjected to one-way analysis of variance (ANOVA) followed by a Tukey's post hoc test (SPSS 17.0 software, Chicago, IL, USA). Results are given as mean \pm S.E. $(n=3)$. Different lowercase letters indicate significant differences from the corresponding control $(p<0.05)$. Dual-luciferase reporter data were subjected to a t-test, and the results are given as mean \pm S.E. $(n=3)$. The level of statistical significance is shown as $p<0.05\left(^{*}\right)$ or $p<0.01\left(^{* *}\right)$.

\section{Results}

\subsection{Sequence Characterization of AjCA and Target Identification of miR-200-3p}

The full-length cDNA of AjCA contained 3029 bp, including a 472-bp 5'UTR and a 1,213-bp 3'UTR. The open reading frame (ORF) contained $1344 \mathrm{bp}$ and encoded a polypeptide of 447 amino acid residues with a calculated molecular mass of $50.55 \mathrm{kDa}$ (Figure 1). InterPro analysis indicated that 
the AjCA protein contained Cyclin_N2, Cyclin_N and Cyclin_C domains (Figure 1, Gene accession No. MN055598). Analysis of the putative miR-200-3p binding sites was then performed using TargetScan 5.2 and Miranda 3.3a programs. Results showed that the binding site started from 2,173 bp to 2,179 bp within the 3'UTR of $A j C A$. Importantly, the binding site was identified and found to be conserved in other marine invertebrate groups: the hood coral Stylophora pistillata and giant owl limpet Lottia gigantea (Figure 2). The theoretical minimum free energy of binding $(\mathrm{kcal} / \mathrm{mol})$ was also calculated using the Miranda program. The theoretical prediction of the thermodynamic binding parameters for miRNA 200-3p binding to $A j C A$ was calculated to be $-10.94 \mathrm{kcal} / \mathrm{mol}$ (below the given theoretical parameters $-10 \mathrm{kcal} / \mathrm{mol}$ ), suggesting that $A j C A$ is a likely target of miR-200-3p in the sea cucumber (Figure 2).

\subsection{The Expression of miR-200-3p and AjCA Gene and the Production of AjCA Protein during the Whole} Period of Aestivation

The expression level of miR-200-3p was significantly up-regulated in the intestine by $2.11 \pm$ 0.47 -fold during the DA (deep aestivation) stage as compared with the NA (non-aestivating) stage $(p<0.05)$, but was down-regulated again in the AA (arousal from aestivation) stage (Figure 3). Both the transcript levels of $A j C A$ and protein levels of AjCA decreased significantly in intestine at the DA stage to $45 \pm 16 \%$ and $71 \pm 1 \%$, respectively, of the corresponding values during the NA stage $(p<0.05)$. However, in the AA stage (Figure $4 \mathrm{~A}, \mathrm{~B}), A j C A$ transcript levels remained low (similar to DA), whereas AjCA protein productions rose to a level slightly higher than (but not significantly different from) the NA value. The expression of $A j C A$ was negatively correlated with the expression of miR-200-3p in the intestine of $A$. japonicus during aestivation.

\subsection{Validation of the Interaction between the 3'UTR of AjCA and miR-200-3p by Dual-Luciferase Reporter} Assays

The interaction of binding sites on $A j C A$ and miR-200-3p was verified by dual-luciferase reporter assays. Information concerning the binding and mutation sites of miR-200-3p in the $3^{\prime} \mathrm{UTR}$ of $A j C A$ is shown in Figure 5A. Compared with the negative control (NC), which lacked co-transfected miRNA, cells with transfected miR-200-3p mimics showed a significant reduction in luciferase reporter activity to $47 \pm 4 \%$ of the NC value $(p<0.01$ ) (Figure $5 \mathrm{~B}$ ), indicating that cells without the co-transfected microRNA were able to express greater amounts of $A j C A$ transcripts. Cells containing a mutated (Mut) segment of the AjCA 3'UTR also showed a significant, but less pronounced, reduction in luciferase reporter activity, to about $70 \%$ of the NC value, suggesting that miR-200-3p was less able to interact with the mutant $A j C A 3^{\prime} \mathrm{UTR}$. 
1 attgtttgtattatatatatattttggetcgaagtctaaaccgettgtacacccattccgttgaggtcagccegttgtaatttctgtggaacccatctggatgca 106 ctaggaatttcatgaatttttatttctttcgttttcacatatattgcaagctgagtatcgttggaatcttttgaagtcagctacaatgaacttttctttcagcgc 211 caaagtggactattgaggegccaagtacatacagtacgtagaccaatggaacgttcagttcag tat taacgggttcgagtaactgcagcacgtaaaagtatagcc 316 ttccaaccttgttctatactatat tatcacatacacgccaaagtactagtaacagtaaagtacat tagactagagaaggcttaaccaccattgcttcaacgtttg 1 M S F V S E E E Y P M Y E S T N L N H 421 ccaaagtctatctttttctgtaagccaatcctccagcggtgttaccagagcgATGTCTTTTGTATCCGAGGAATACCCCATGTATGAAAGCACAAACCTGAATCA $\begin{array}{llllllllllllllllllllllllllllllllllll}19 & N & S & A & L & Q & Q & T & R & K & T & K & R & E & E & T & T & I & R & G & N & G & P & Q & A & C & K & R & P & A & L & G & I & I & T & N\end{array}$ 526 TAACTCTGCACTTCAACAAACGAGGAAAACCAAAAGAGAAGAAACAACGATAAGGGGAAATGGACCTCAGGCATGCAAACGTCCCGCTCTTGGCATTATAACCAA $\begin{array}{lllllllllllllllllllllllllllllllllllll}54 & N & V & G & R & V & Q & P & A & R & A & A & K & Q & A & T & S & S & G & R & F & N & S & N & S & E & N & V & P & P & S & F & D & G & C & Q\end{array}$ 631 TAATGTTGGGCGCGTCCAGCCTGCAAGAGCAGCAAAACAGGCAACTTCTTCTGGGAGATTTAACAGCAATTCTGAGAATGTTCCACCTAGCTTTGACGGTTGTCA $\begin{array}{llllllllllllllllllllllllllllllllllll}89 & \text { K } & S & N & G & F & V & F & P & G & S & S & T & D & L & G & F & T & I & H & V & D & P & I & E & S & R & L & G & H & S & T & N & V & N & S\end{array}$ 736 AAAGTCCAATGGATTTGTATTCCCAGGGTCATCTACAGATCTTGGCTTTACCATCCATGTAGATCCAATCGAAAGCCGTCTTGGTCATTCTACAAACGTCAACTC $\begin{array}{lllllllllllllllllllllllllllllllllllll}124 & Q & T & E & N & E & T & S & L & L & N & P & A & V & T & A & L & P & R & P & P & L & T & C & L & E & N & V & D & I & D & L & E & P & S & P\end{array}$ 841 CCAGACAGAAAATGAGACGTCCCTTTTAAATCCAGCTGTGACTGCACTACCCCGACCACCGTTAACATGTCTGGAAAATGTGGACATCGACTTAGAACCGTCTCC $\begin{array}{llllllllllllllllllllllllllllllllllll}159 & M & V & L & D & T & S & L & T & E & N & Q & L & H & S & Q & T & D & R & E & T & D & R & K & D & S & I & Y & E & V & S & E & Y & A & E & E\end{array}$ 946 TATGGTCCTAGATACATCACTGACAGAGAACCAGTTACATTCACAGACGGACAGAGAAACAGACCGCAAGGACAGTATTTATGAAGTCTCGGAATACGCAGAGGA $\begin{array}{llllllllllllllllllllllllllllllllllll}194 & \text { I } & F & Q & Y & L & R & E & A & E & L & R & N & R & P & K & A & G & Y & M & K & K & Q & P & D & I & T & A & S & M & R & C & I & L & V & D\end{array}$ 1051 GATTTTCCAATATTTACGGGAAGCTGAGCTCCGAAACAGACCCAAAGCAGGTTACATGAAGAAACAGCCAGACATCACTGCTAGTATGAGGTGTATCTTGGTCGA 229 WI L 1156 TTGGTTGGTGGAAGTTGCCGAAGAGTATAAATTAGCCAATGAAACGCTCTACCTGGCAGTCTCTTATATAGATCGGTTCCTCTCTCACATGAGCGTATTAAGAGC

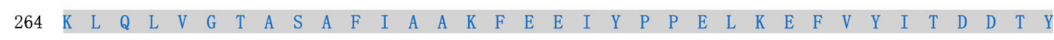
1261 CAAACTTCAGCTGGTCGGAACGGCAAGTGCATTCATAGCAGCTAAATTTGAGGAGATTTATCCGCCAGAGTTGAAGGAATTTGTTTACATCACAGACGATACATA 299 N S S K 1366 TAATTCCAAGCAGGTCTTACGGATGGAACACATGATTCTGAAAGTACTGTCCTTTGATCTGGCTGTCCCGACAATCAACGTTTTCCTACCCCGGTATATCAAGGC 334

1471 CAGCGACGAAGGAGTCGACGCAAGATTGGGAGACTTAACGAAGTATTTGGCAGAACTCTCCTTGCAACATTTTGAGTTTGTAAAGTACCTTCCTTCTATGgTAGC 369

1576 TGCTTCTGCAACCTGTCTGGCTCGACACACATTGCATCTCCATGCTTGGACGCCCCAACTATTCCACGCAACCACTTATCAGTTTGAAGACATCGAGGATTGTGT 404 1681 CGGGGAGTTACATCATGTTTTCAAAGAAGCACCCAGTTTCCCTCAGCAGGCAGTACGAGATAAATACAAGTCACAAAAGTACAACAACATATCGCTGACTACAGT 439 管 1786 CCGCGAGGATGTTCCCACCCTTTGCTCATAGgacatctccaaat taaaggaaggggagacatt ttacaatcatgtcagaggtgatgatatt tactctcagcaaat 1891 caattgaccatgccaaattgatttttaagtgacgtaaagaactgtttgacaagctttaaaagttgaaaccagtgctacaaacgctgettgaaatgaccaaacgtt 1996 aaatgaatgcatatcacaacacgtatctggttgctacgagatgaccctcctgtgtctgtaaccgggggtgtccaccatttttatcaaaggtaataaagtagtccg 2101 ttgatgctgaaacaagatgacttatagggggtggttccttcaacataggaatgcgtttttaaaatgaatcttcagtattacaagaacgggtttttaccggacagt 2206 tacggeccgttgcattaaatacgccaatttgagacaatcggaacgccactttggtgtttttatagtccttttaaaatttctaaaacatttttaaaatgetgttga 2311 agattttacagtagtagttttaaatgtcgttttaatgtcagtcatgttttataacatgtttaaaaaagaaatgttgaaatagaagaaatctaaatgaagagtga 2416 aattcccaggccacaaaaagaataggtaagacagcttttcactctgtattttaaat taaatttcgaagagccagaatgattttaaccatggcatactaataacca 2521 tgagtgaaacatgcagtatgtaaatctgtgtaaccattgatcaacggtcattgtccgatttttgttgtgatgtcactgccacctgtgttctgtactcaaacggtt 2626 tgattagccaattaggggtagttatcaaagttataaatattcatgagattggctccttgaccttcttgatgcagaaatactaggtttacctcctttttagttttc 2731 aattatgaggtaacaatttaaactactacttgagat tat ttgtggtgtcgacaaatcaatcatgaattgectactttcgtctcggetaggtttttattgtattca 2836 tgtggttttacaatgtcgttggtttgtcgataggacttttatgttatgtatatcaacatttatcctctttttatttcacaacaaatagacaaacag taagaggaa 2941 ttattttcaaccacttttcaagtctctgtagataagttcaatcctgtcttaaagaccatcttaacttttcatttgtacataagtgttac

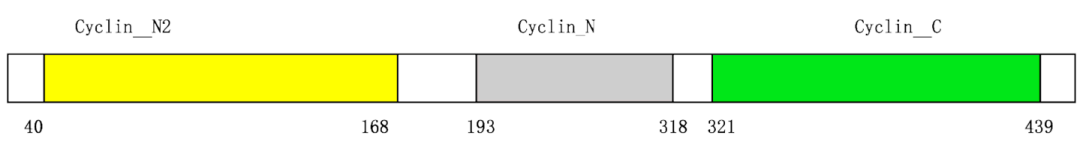

Figure 1. The complete cDNA sequence and deduced amino acid sequence of $A j C A$. Coding and noncoding regions are shown by uppercase and lowercase letters, respectively. The asterisk indicates the translational termination codon. At the bottom of the page is the schematic diagram of domains and characteristic motifs. 
A

\begin{tabular}{|c|c|c|}
\hline 5'UTR & ORF & 3'UTR \\
\hline
\end{tabular}

Apostichopus japonicus 5 ' ...TAAAATGAATCTTCAGTATTACAAGAACGGGTTT...3'

Stylophora pistillata 5 , ...TTTTTTTAACTTCTCAGTATTACAAAGCTGTACAG...3'

Lottia gigantea $\quad 5$, ...GAGTTGTGAGTGACAGTATTTTGAAAGGTGTGTG...3'

B $\quad$ miR-200-3p

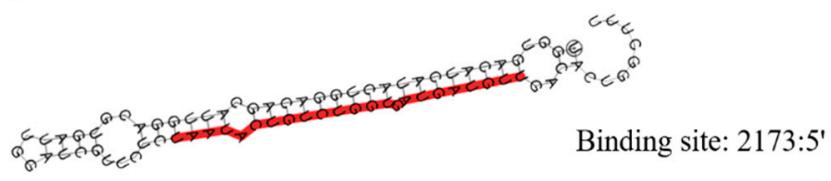

$\mathrm{mfe}:-10.94 \mathrm{kcal} / \mathrm{mol}$

Figure 2. Theoretical binding of miR-200-3p to a conserved region in the $3^{\prime} \mathrm{UTR}$ of the $A j C A$ gene. (A) Conservation analysis of the miR-200-3p binding site in the Cyclin $A$ gene from the sea cucumber $A$. japonicus, hood coral Stylophora pistillata and giant owl limpet Lottia gigantea. (B) Predicted binding structure of miR-200-3p when binding to the 3'UTR of $A j C A$ and the mature miR-200-3p sequence (shown in red), as determined from TargetScan and miRanda programs.

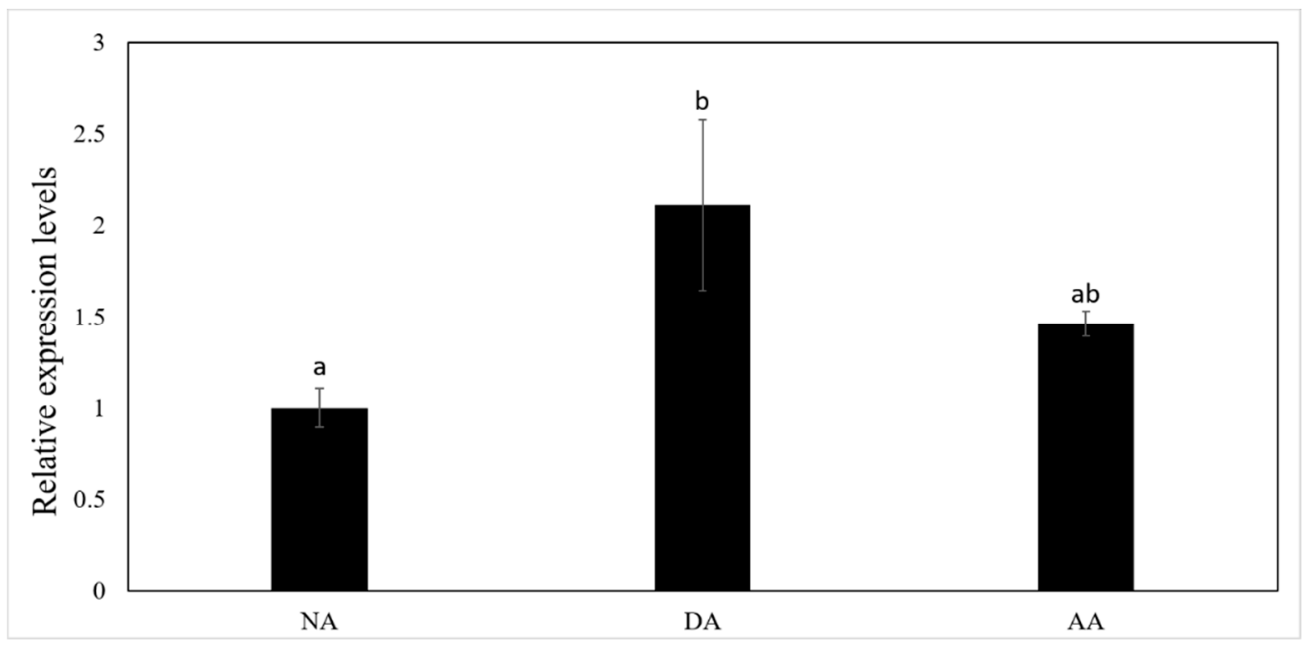

Figure 3. The relative expression of miR-200-3p in the intestine of $A$. japonicus at non-aestivating (NA), deep-aestivation (DA), and arousal from aestivation (AA) stages. The expression of miR-200-3p was detected by qRT-PCR in the intestine of $A$. japonicus from NA, DA and AA groups. Data are means $\pm \mathrm{SE}$ ( $n=5$ independent trials on tissue from different animals). Different lowercase letters indicate groups that are significantly different from each other $(p<0.05)$. 


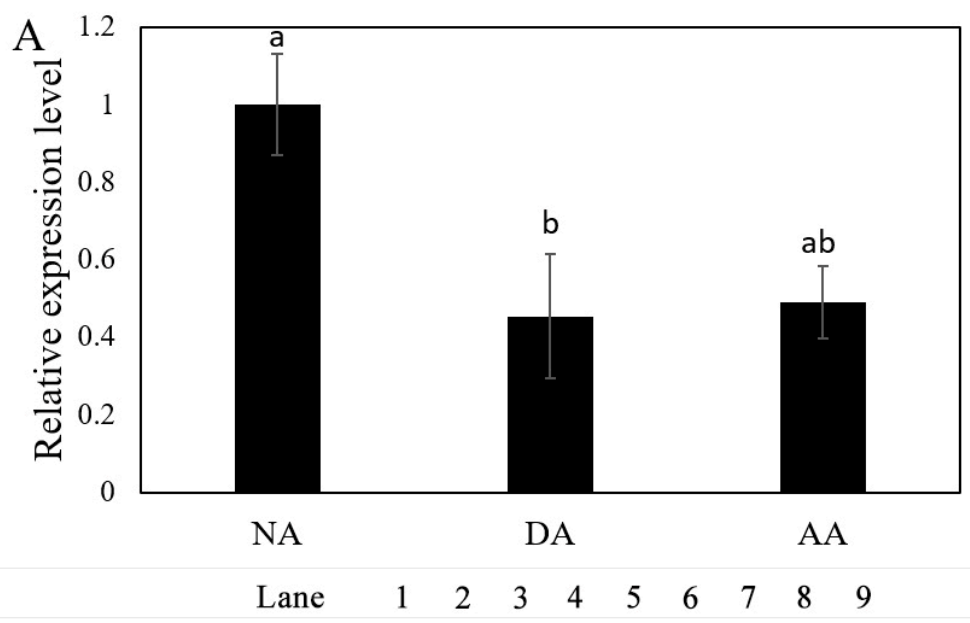

B Cyclin A $50 \mathrm{kDa}$

$\beta$-tubulin $55 \mathrm{kDa}$

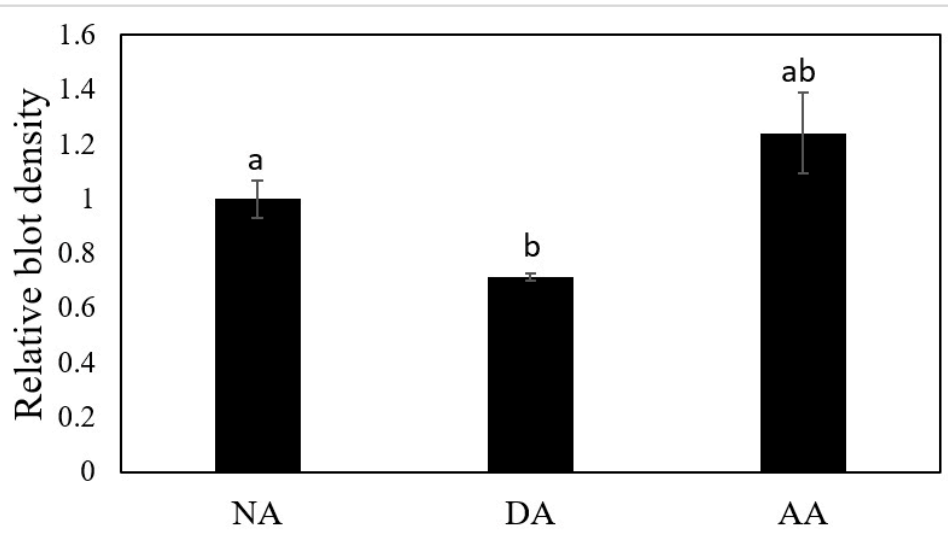

Figure 4. The mRNA expression and protein production levels of $A j C A$ in the intestine of $A$. japonicus at NA, DA and AA stages. (A) Relative mRNA expression levels of $A j C A$ in the intestine of NA, DA and AA groups, determined by qRT-PCR. Values were standardized against $\beta$-tubulin and $\beta$-actin. Values are means \pm SE $(n=5)$. Different lowercase letters indicate groups that are significantly different from each other $(p<0.05)$. (B) Relative protein levels of AjCA at the NA, DA and AA stages in intestine as determined by Western blot. Representative bands show blot intensities for NA (lanes 1-3), DA (lanes 4-6) and AA (lanes 7-9) groups. AjCA protein levels were standardized against the corresponding $\beta$-tubulin band densities for the same samples. Histograms show the standardized levels for NA, $\mathrm{DA}$ and AA. Values are means $A j C A \pm \mathrm{SE}(n=3)$. Different lowercase letters indicate groups that are significantly different from each other $(p<0.05)$. 


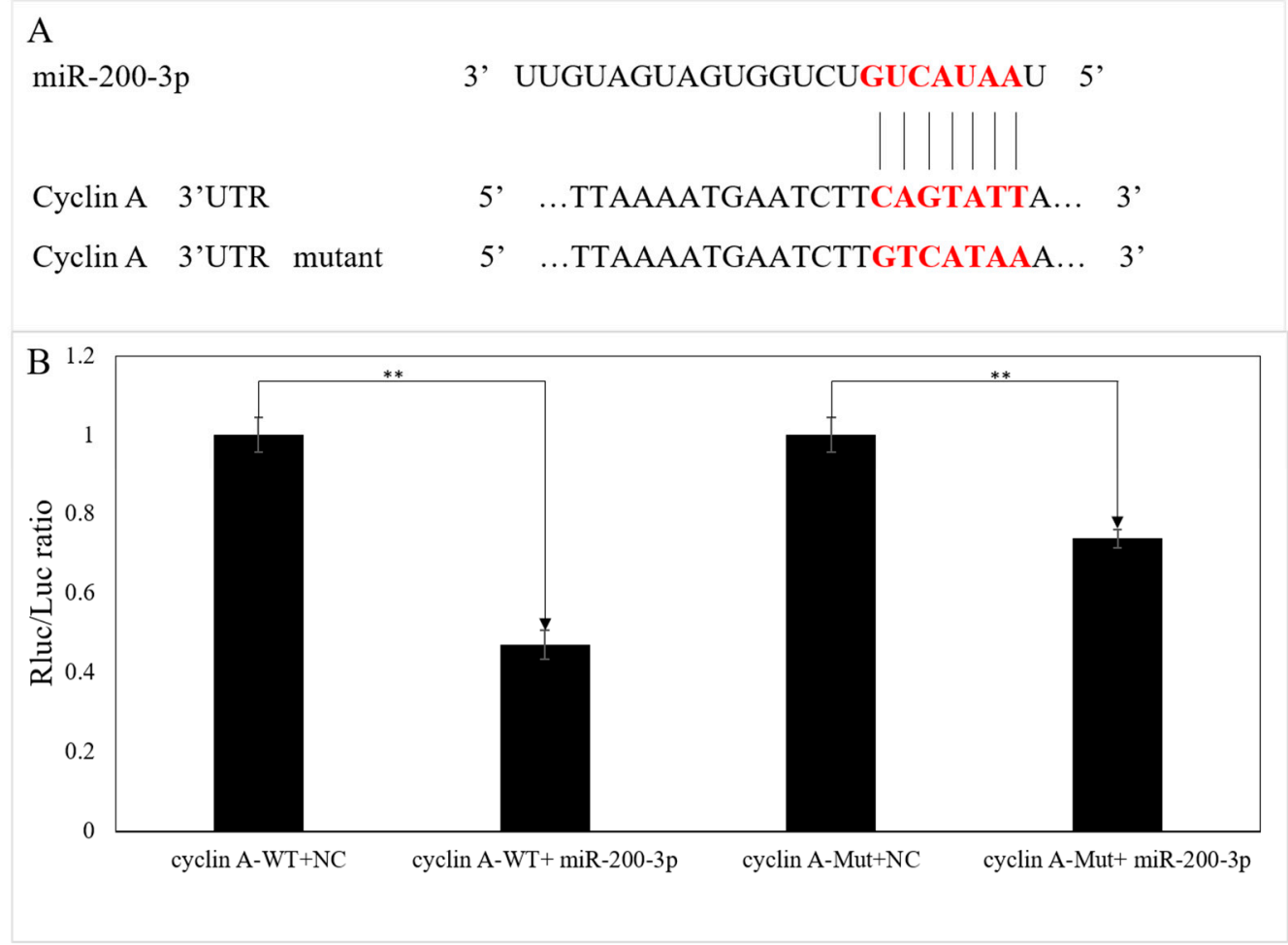

Figure 5. Validation of the binding sites between miR-200-3p and $3^{\prime} U T R$ of $A j C A$. (A) Schematic representation of the putative miRNA-200-3p targeting sites in $A j C A$ mRNA and the respective mutant sites. (B) HEK-293T cells were co-transfected with the pmiR-RB-REPORT ${ }^{\mathrm{TM}}$ vectors, carrying the wild-type (WT) or the mutated (Mut) AjCA 3'-UTR, pRLCMV-Renilla-luciferase, and control miR-200-3p mimics as indicated. ${ }^{* *}$ indicates a significant difference $(p<0.01)$. NC: negative control without miR-200-3p.

\subsection{Gain and Loss of Function Analysis of miR-200-3p In Vivo}

The miR-200-3p targeted regulation of $A j C A$ was further verified in vivo using $A$. japonicus samples that were transfected with miR-200-3p mimics, or a miR-200-3p inhibitor, or their negative controls (sequences of all are shown in Table 1). As shown in Figure 6, the overexpression of miR-200-3p mimics significantly decreased both the transcript and protein levels of $A j C A$ by about $60 \%$ in intestine tissue as compared with the negative control group $(p<0.05)$ (Figure 6A,B). Furthermore, the opposite occurred when the expression of miR-200-3p was inhibited. A significant increase in $A j C A$ transcripts of nearly 3-fold occurred, and protein levels increased by about 1.4-fold in A. japonicus intestine tissue $(p<0.01$ and $p<0.05$, respectively, Figure $6 \mathrm{~A}, \mathrm{~B})$. 

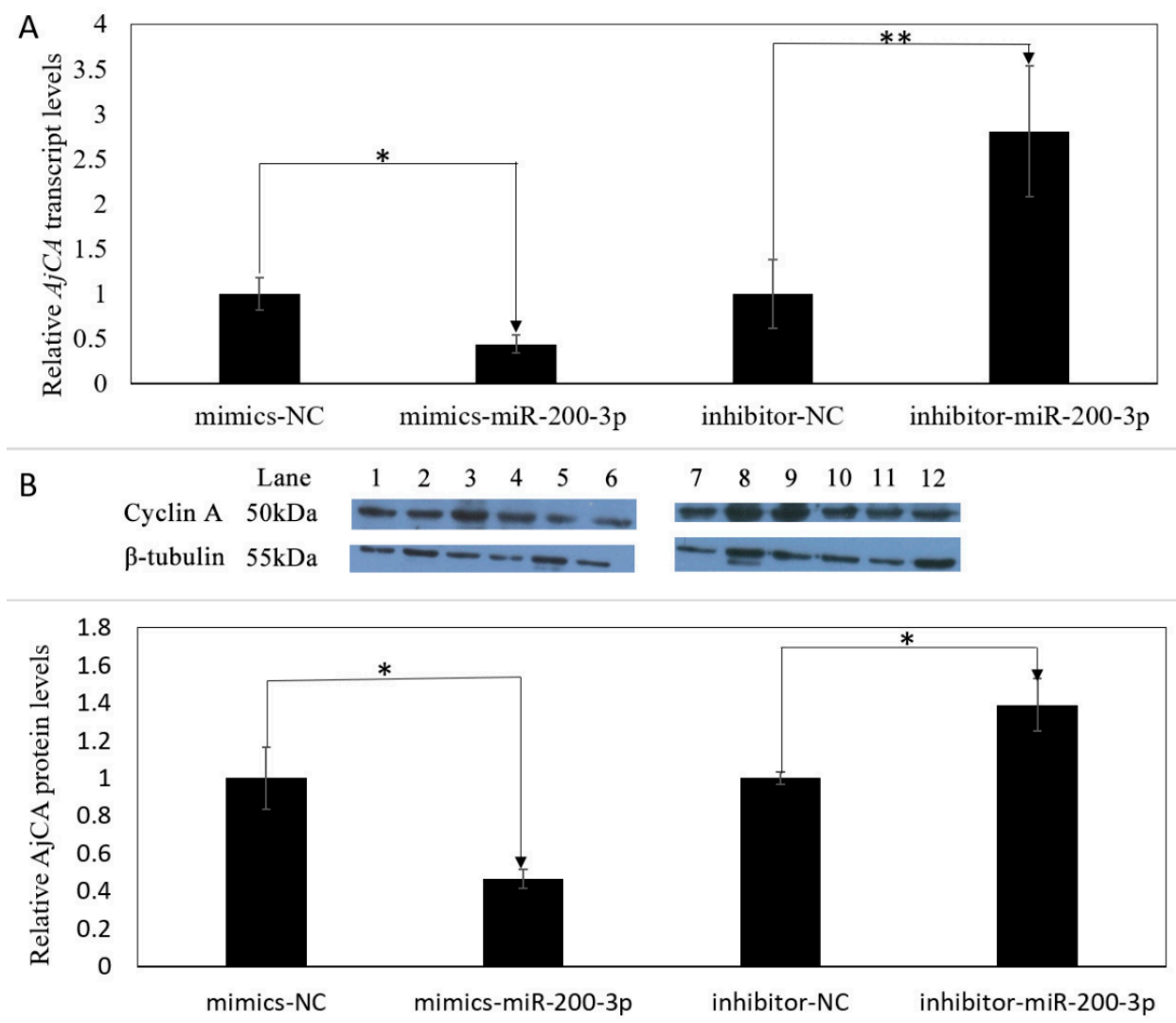

Figure 6. Gain and loss of function analysis of miR-200-3p in the intestine of A. japonicus in vivo. (A) Relative $A j C A$ transcript levels after transfection with miR-200-3p mimics or inhibitor. Values were normalized against $\beta$-tubulin and $\beta$-actin. Values are means $\pm \operatorname{SE}(n=5)$. ${ }^{*}$ indicates a significant difference $(p<0.05) ;{ }^{* *}(p<0.01)$. (B) Relative AjCA protein production after transfection with miRNA mimics or inhibitor. Representative bands show blot intensity. Lanes show the treatments as follows: (1-3) miR-200-3p mimics, negative control; (4-6) miR-200-3p mimics; (7-9) miR-200-3p inhibitor, negative control; (10-12) miR-200-3p inhibitor. Corresponding tubulin bands are also shown. Values were standardized against the corresponding densities for $\beta$-tubulin. Values are means $\pm \mathrm{SE}(n=3)$. * indicates a significant difference $(p<0.05)$.

\section{Discussion}

The cell cycle is the major process responsible for regulating cell division and tissue growth and is a highly resource- and energy-expensive process [32]. The regulation of the cycle depends on the sequential expression of key cyclins during different phases of the cycle. Cyclin A is a core and particularly interesting regulator that activates two different cyclin-dependent kinases (Cdk1 and Cdk2) and functions in both the $S$ phase and in mitosis [33]. It has been reported that inactivation of cyclin A in Drosophila or mammalian cultured cells arrests the cell cycle in the G2 phase [34]. Cell cycle suppression/arrest is known to be part of hypometabolism in animals that use dormancy or torpor to survive during times of extended environmental stress. Control over cyclins occurs at transcriptional and translational levels as well as by epigenetic mechanisms, such as control over cyclin D1 during anoxia exposure in turtles by microRNA inhibition of mRNA translation [35] and cyclin B arrest by DNA methylation in aestivating sea cucumbers [10]. In our present study, the expression patterns of $A j C A$ transcripts and AjCA protein were tracked over the aestivation-arousal cycle. Based on this, we hypothesized that one or more specific control mechanisms, focused on $A j C A$, may probably be central to global cell cycle arrest during sea cucumber aestivation.

Research over the last decade has demonstrated that miRNAs play integral roles in metabolic rate depression in multiple animal systems, where they help to modulate the flow of mRNA transcripts to 
one of three fates: immediate availability for translation, storage for later translation, or degradation. By modulating these choices, miRNA action can be a powerful, yet metabolically simple, means of altering global energy expenditure on protein synthesis that is readily adaptable for use in coordinating reversible transitions to and from hypometabolic states. The miR-200 family is one of the best-known miRNA families in mammals [36]. As a member of this family, miR-200-3p has been reported to play roles in cell proliferation, apoptosis and tumorigenesis [37,38]. However, focused studies of miR-200-3p effects within metabolically or environmentally stressed cells are still very limited. In our present study, $A j C A$ was putatively identified as a novel target of miR-200-3p using bioinformatics analysis combining TargetScan 5.2 and Miranda 3.3a software, and a negative relationship between miR-200-3p and AjCA expression at both transcriptional and translational levels was documented. This correlation suggests that miRNA-200-3p interacted with $A j C A$ transcripts at a post-transcriptional level-i.e., diverting transcripts away from the transcriptional apparatus and into degradation or perhaps into storage granules or P-bodies during aestivation. This led to reduced synthesis of AjCA protein. In the deep aestivation (DA) situation, the above effects may also be integrated with other controls that operate at a global level to inhibit overall transcription and translation of all cell cycle proteins during torpor. This is probably needed in the intestine due to its high proliferative potential that must be strongly suppressed during the months of nonfeeding [39]. Therefore, we further investigated miR-200-3p regulation of $A j C A$. Using dual-luciferase reporter assays, the results confirmed that the binding site predicted between miR-200-3p and $A j C A$ is the potential targeted location. This binding site was also conserved in other marine invertebrate groups (e.g., hood coral Stylophora pistillata and giant owl limpet Lottia gigantea), suggesting that cyclin $A$ is probably a common gene target of miR-200-3p in marine invertebrates.

To further support our claims, gain- and loss-of-function experiments were conducted in vivo to verify the interaction between miR-200-3p and AjCA by qRT-PCR and Western blotting. The results showed that overexpression of miR-200-3p negatively influenced $A j C A$ expression at both at the transcriptional and translational levels in A. japonicus intestine. Oppositely, inhibition of miR-200-3p led to increased levels of $A j C A$ transcripts and AjCA protein. Hence, we propose that miR-200-3p acts as a regulatory mechanism affecting cyclin A and may also affect other cell cycle components when sea cucumbers enter aestivation. Indeed, the inhibition of cyclin A has been confirmed as one of the conserved and general strategies of environmental stress-induced hypometabolism in other species [16].

In summary, this study provides the first demonstration of $A j C A$ regulation in aestivating sea cucumbers, A. japonicus, and suggests that aestivation-responsive suppression of cyclin A transcripts and protein in the intestine are linked to the actions of microRNA. Additionally, miR-200-3p likely has an influence on the overall proliferation state of cells during hypometabolism, as it is also known to target other genes involved in cell proliferation and apoptosis, such as the FOXO (forkhead box) transcription factor and its related signaling pathways [40]. Further studies are required to assess the full degree of influence exerted by miR-200-3p on both cyclin A and other gene targets in sea cucumbers and their involvement in aestivation and environmental stress resistance. To date, this is difficult since very few functional studies of miRNAs have been conducted among marine invertebrates. However, the characteristics of microRNA regulation dovetail with the need for mechanisms of environmental stress response to be broadly applicable, readily coordinated, easily induced and readily reversed [41]. We hope our studies will push forward this research field and greatly improve our understanding of this epigenetic mechanism in marine invertebrates.

Author Contributions: M.C. originally conceived the study. S.W. and M.C. contributed to the acquisition of data. S.W., M.C., Y.Y. and K.B.S. contributed to the interpretation of the data. M.C. and S.W. wrote the first draft of the manuscript. All authors contributed to manuscript revision, and read and approved the submitted version.

Funding: This research was funded by National Natural Science Foundation China (grant No. 41676124).

Acknowledgments: This research was supported by a grant (No. 41676124) from the National Natural Science Foundation China. 
Conflicts of Interest: The authors declare no conflict of interest.

\section{References}

1. Liao, Y. Fauna Sinica: Phylum Echinodermata Class Holothuroidea; Science Press: Beijing, China, 1997; pp. 148-150. (In Chinese)

2. Yang, H.; Zhou, Y.; Zhang, T.; Yuan, X.; Li, X.; Liu, Y.; Zhang, F. Metabolic characteristics of sea cucumber Apostichopus japonicus (Selenka) during aestivation. J. Exp. Mar. Biol. Ecol. 2006, 330, 505-510. [CrossRef]

3. Yuan, X.; Yang, H.; Chen, M.; Gao, F. Research advances in aestivation of sea cucumber Apostichopus japonicus (Selenka): A review. Mar. Sci. 2007, 31, 88-90.

4. Gao, F.; Yang, H.; Xu, Q.; Wang, F.; Liu, G.; German, D.P. Phenotypic plasticity of gut structure and function during periods of inactivity in Apostichopus japonicus. Comp. Biochem. Physiol. B Biochem. Mol. Biol. 2008, 150, 255-262. [CrossRef] [PubMed]

5. Chen, M.; Zhang, X.; Liu, J.; Storey, K.B. High-throughput sequencing reveals differential expression of miRNAs in intestine from sea cucumber during aestivation. PLoS ONE 2013, 8, e76120. [CrossRef] [PubMed]

6. Zhao, Y.; Yang, H.S.; Storey, K.B.; Chen, M.Y. RNA-Seq dependent transcriptional analysis unveils gene expression profile in the intestine of sea cucumber Apostichopus japonicus during aestivation, Comp. Biochem. Physiol. D 2014, 10, 30-43.

7. Chen, M.; Storey, K.B. Large-scale identification and comparative analysis of miRNA expression profile in the respiratory tree of the sea cucumber Apostichopus japonicus during aestivation. Mar. Genom. 2014, 13, 39-44. [CrossRef] [PubMed]

8. Chen, M.; Li, X.; Zhu, A.; Storey, K.B.; Sun, L.; Gao, T.; Wang, T. Understanding mechanism of sea cucumber Apostichopus japonicus aestivation: Insights from TMT-based proteomic study. Comp. Biochem. Physiol. Part D Genom. Proteom. 2016, 19, 78-89. [CrossRef]

9. Chen, M.; Zhu, A.; Storey, K.B. Comparative phosphoproteomic analysis of intestinal phosphorylated proteins in active versus aestivating sea cucumbers. J. Proteom. 2016, 135, 141-150. [CrossRef]

10. Zhu, A.; Chen, M.; Zhang, X.; Storey, K.B. Gene structure, expression, and DNA methylation characteristics of sea cucumber cyclin B gene during aestivation. Gene 2016, 594, 82-88. [CrossRef]

11. Chen, M.; Wang, S.; Li, X.; Storey, K.B.; Zhang, X. The potential contribution of miRNA-200-3p to the fatty acid metabolism by regulating AjEHHADH during aestivation in sea cucumber. PeerJ 2018, 6, e5703. [CrossRef]

12. Storey, K.B.; Storey, J.M. Putting life on 'pause'-Molecular regulation of hypometabolism. J. Exp. Biol. 2007, 210, 1700-1714. [CrossRef] [PubMed]

13. Ambros, V. The C. elegans heterochronic gene lin- 4 encodes small RNAs with antisense complementarity to lin-14. Cell 1993, 75, 843-854.

14. Biggar, K.K.; Storey, K.B. Functional impact of microRNA regulation in models of extreme stress adaptation. J. Mol. Cell Biol. 2018, 10, 93-101. [CrossRef] [PubMed]

15. Liu, Y.; Hu, W.; Wang, H.; Lu, M.; Shao, C.; Menzel, C.; Yan, Z.; Li, Y.; Zhao, S.; Khaitovich, P.; et al. Genomic analysis of miRNAs in an extreme mammalian hibernator, the Arctic ground squirrel. Physiol. Genom. 2010, 42, 39-51. [CrossRef] [PubMed]

16. Biggar, K.K.; Storey, K.B. The emerging roles of microRNAs in the molecular responses of metabolic rate depression. J. Mol. Cell Biol. 2011, 3, 167-175. [CrossRef] [PubMed]

17. Kornfeld, S.F.; Biggar, K.K.; Storey, K.B. Differential expression of mature microRNAs involved in muscle maintenance of hibernating little brown bats, Myotis lucifugus: A model of muscle atrophy resistance. Genom. Proteom. Bioinform. 2012, 10, 295-301. [CrossRef] [PubMed]

18. Biggar, K.K.; Storey, K.B. Identification and expression of microRNA in the brain of hibernating bats, Myotis lucifugus. Gene 2014, 544, 67-74. [CrossRef] [PubMed]

19. Lang-Ouellette, D.; Morin, P.J. Differential expression of miRNAs with metabolic implications in hibernating thirteen-lined ground squirrels, Ictidomys tridecemlineatus. Mol. Cell. Biochem. 2014, 394, 291-298. [CrossRef] [PubMed]

20. Luu, B.E.; Biggar, K.K.; Wu, C.W.; Storey, K.B. Torpor-responsive expression of novel microRNAs regulating metabolism and other cellular pathways in the thirteen-lined ground squirrel, Ictidomys tridecemlineatus. FEBS Lett. 2016, 590, 3574-3582. [CrossRef] [PubMed] 
21. Hadj-Moussa, H.; Moggridge, J.A.; Luu, B.E.; Quintero-Galvis, J.F.; Gaitán-Espitia, J.D.; Nespolo, R.F.; Storey, K.B. The hibernating South American marsupial, Dromiciops gliroides, displays torpor-sensitive microRNA expression patterns. Sci. Rep. 2016, 6, 24627. [CrossRef]

22. Biggar, K.K.; Storey, K.B. Perspectives in cell cycle regulation: Lessons from an anoxic vertebrate. Curr. Genom. 2009, 10, 573-584. [CrossRef] [PubMed]

23. Wu, C.W.; Storey, K.B. Pattern of cellular quiescence over the hibernation cycle in liver of thirteen-lined ground squirrels. Cell Cycle 2012, 11, 1714-1726. [CrossRef] [PubMed]

24. Swenson, K.; Farrell, K.M.; Ruderman, J.V. The clam embryo protein cyclin A induces entry into M phase and the resumption of meiosis in Xenopus oocytes. Cell 1986, 47, 861-870. [CrossRef]

25. Kanakkanthara, A.; Jeganathan, K.B.; Limzerwala, J.F.; Baker, D.J.; Hamada, M.; Nam, H.J.; Deursen, W.H.; Hamada, N.; Naylor, R.M.; Becker, N.A.; et al. Cyclin A2 is an RNA binding protein that controls Mre11 mRNA translation. Science 2016, 353, 1549-1552. [CrossRef] [PubMed]

26. Barlat, I.; Fesquet, D.; Brechot, C.; Henglein, B.; Dangeac, A.D.; Vie, A.; Blanchard, J.M. Loss of the G1-S control of cyclin A expression during tumoral progression of Chinese hamster lung fibroblasts. Cell Growth Differ. 1993, 4, 105-113. [PubMed]

27. Gramantieri, L.; Ferracin, M.; Fornari, F.; Veronese, A.; Sabbioni, S.; Liu, C.G.; Calin, G.A.; Giovannini, C.; Ferrazzi, E.; Grazi, G.L.; et al. Cyclin G1 is a target of miR-122a, a microRNA frequently down-regulated in human hepatocellular carcinoma. Cancer Res. 2007, 67, 6092-6099. [CrossRef] [PubMed]

28. Wang, W.; Zhao, L.J.; Tan, Y.X.; Ren, H.; Qi, Z.T. MiR-138 induces cell cycle arrest by targeting cyclin D3 in hepatocellular carcinoma. Carcinogenesis 2012, 33, 1113-1120. [CrossRef] [PubMed]

29. Zhang, P.; Zheng, C.; Ye, H.; Teng, Y.; Zheng, B.; Yang, X.; Zhang, J. MicroRNA-365 inhibits vascular smooth muscle cell proliferation through targeting cyclin D1. Int. J. Med. Sci. 2014, 11, 765-770. [CrossRef]

30. Lines, K.E.; Newey, P.J.; Yates, C.J.; Stevenson, M.; Dyar, R.; Walls, G.V.; Bowl, M.R.; Thakker, R.V. miR-15a/miR-16-1 expression inversely correlates with cyclin D1 levels in Men1 pituitary NETs. J. Endourol. 2018, 240, 41-50. [CrossRef]

31. Wang, S.S.; Li, X.K.; Chen, M.Y.; Storey, K.B.; Wang, T.M. A potential antiapoptotic regulation: The interaction of heat shock protein 70 and apoptosis-inducing factor mitochondrial 1 during heat stress and aestivation in sea cucumber. J. Exp. Zool. A Ecol. Integr. Physiol. 2018, 329, 103-111. [CrossRef]

32. Obaya, A.J.; Sedivy, J.M. Regulation of cyclin-Cdk activity in mammalian cells. Cell. Mol. Life Sci. 2002, 59, 126-142. [CrossRef] [PubMed]

33. Pagano, M.; Pepperkok, R.; Verde, F.; Ansorge, W.; Draetta, G. Cyclin A is required at two points in the human cell cycle. EMBO J. 1992, 11, 961-971. [CrossRef] [PubMed]

34. Thomas, D.P.; William, C.E.; Jennifer, L.; Graham, J. Cell Biology, 3rd ed.; Elsevier: Philadelphia, PA, USA, 2017.

35. Biggar, K.K.; Storey, K.B. Evidence for cell cycle suppression and microRNA regulation of cyclin D1 during anoxia exposure in turtles. Cell Cycle 2012, 11, 1705-1713. [CrossRef] [PubMed]

36. Feng, X.; Wang, Z.; Fillmore, R.; Xi, Y. MiR-200, a new star miRNA in human cancer. Cancer Lett. 2014, 344, 166-173. [CrossRef] [PubMed]

37. Pautke, C.; Schieker, M.; Tischer, T.; Kolk, A.; Neth, P.; Mutschler, W.; Milz, S. Characterization of osteosarcoma cell lines MG-63, Saos-2 and U-2 OS in comparison to human osteoblasts. Anticancer Res. 2004, 24, 3743-3748. [PubMed]

38. Xiao, Y.; Yan, W.; Lu, L.; Wang, Y.; Lu, W.; Cao, Y.; Cai, W. p38/p53/miR-200a-3p feedback loop promotes oxidative stress-mediated liver cell death. Cell Cycle. 2015, 14, 1548-1558. [CrossRef] [PubMed]

39. Su, L.; Chen, M.; Wang, T.; Liu, J.; Zhao, Y.; Yang, H. The division of different stages of aestivation and quantitative analysis of tissue layers thickness of anterior intestine in sea cucumber Apostichopus japonicus. Mar. Sci. 2012, 36, 1-5.

40. Li, Y.; Wu, Y. MiR-200-3p inhibits tumor cell proliferation and induces apoptosis by upregulation of FOXO1 in osteosarcoma cells. Mol. Cell. Toxicol. 2018, 14, 73-78. [CrossRef]

41. Storey, K.B.; Storey, J.M. Metabolic rate depression in animals: Transcriptional and translational controls. Biol. Rev. Camb. Philos. Soc. 2004, 79, 207-233. [CrossRef] [PubMed] 